\title{
Sorafenib in advanced melanoma: a Phase II randomised discontinuation trial analysis
}

\author{
T Eisen*,I, T Ahmad', KT Flaherty², M Gore', S Kaye', R Marais', I Gibbens', S Hackett', M James', \\ LM Schuchter ${ }^{2}$, KL Nathanson ${ }^{2}, \mathbf{C ~ X i a}^{3}$, R Simantov $^{3}$, B Schwartz $^{3}$, M Poulin-Costello $^{3}$, PJ O'Dwyer $^{2}$ \\ and MJ Ratain ${ }^{4}$
}

'Royal Marsden Hospital, Downs Road, Surrey SMT 5PT, UK; ${ }^{2}$ Abramson Cancer Center of the University of Pennsylvania, Philadelphia, PA 19104, USA;

${ }^{3}$ Bayer Pharmaceuticals Corporation, West Haven, CT 065I6, USA; ${ }^{4}$ Department of Medicine, University of Chicago, Chicago, IL 60637, USA

\begin{abstract}
The effects of sorafenib - an oral multikinase inhibitor targeting the tumour and tumour vasculature - were evaluated in patients with advanced melanoma enrolled in a large multidisease Phase II randomised discontinuation trial (RDT). Enrolled patients received a I2-week run-in of sorafenib $400 \mathrm{mg}$ twice daily (b.i.d.). Patients with changes in bi-dimensional tumour measurements $<25 \%$ from baseline were then randomised to sorafenib or placebo for a further 12 weeks (ie to week 24 ). Patients with $\geqslant 25 \%$ tumour shrinkage after the run-in continued on open-label sorafenib, whereas those with $\geqslant 25 \%$ tumour growth discontinued treatment. This analysis focussed on secondary RDT end points: changes in bi-dimensional tumour measurements from baseline after 12 weeks and overall tumour responses (WHO criteria) at week 24, progression-free survival (PFS), safety and biomarkers (BRAF, KRAS and NRAS mutational status). Of 37 melanoma patients treated during the run-in phase, 34 were evaluable for response: one had $\geqslant 25 \%$ tumour shrinkage and remained on open-label sorafenib; six (16\%) had $<25 \%$ tumour growth and were randomised (placebo, $n=3$; sorafenib, $n=3$ ); and 27 had $\geqslant 25 \%$ tumour growth and discontinued. All three randomised sorafenib patients progressed by week 24; one remained on sorafenib for symptomatic relief. All three placebo patients progressed by week-24 and were re-started on sorafenib; one experienced disease re-stabilisation. Overall, the confirmed best responses for each of the 37 melanoma patients who received sorafenib were $19 \%$ stable disease (SD) (ie $n=1$ open-label; $n=6$ randomised), $62 \%(n=23)$ progressive disease (PD) and $19 \%(n=7)$ unevaluable. The overall median PFS was I I weeks. The six randomised patients with SD had overall PFS values ranging from 16 to 34 weeks. The most common drug-related adverse events were dermatological (eg rash/desquamation, 5 I\%; hand-foot skin reaction, 35\%). There was no relationship between V600E BRAF status and disease stability. DNA was extracted from the biopsies of $17 / 22$ patients. Six had V600E-positive tumours ( $n=4$ had PD; $n=$ I had SD; $n=$ I unevaluable for response), and I I had tumours containing wild-type BRAF ( $n=9$ PD; $n=1$ SD; $n=1$ unevaluable for response). In conclusion, sorafenib is well tolerated but has little or no antitumour activity in advanced melanoma patients as a single agent at the dose evaluated (400 mg b.i.d.). Ongoing trials in advanced melanoma are evaluating sorafenib combination therapies.
\end{abstract}

British Journal of Cancer (2006) 95, 58I-586. doi: I 0.I038/sj.bjc.660329I www.bjcancer.com

Published online I August 2006

(c) 2006 Cancer Research UK

Keywords: Sorafenib; multikinase inhibitor; advanced melanoma; V600E BRAF; randomised discontinuation trial

The incidence of malignant melanoma is rising, and the current treatment options for patients with metastatic disease are limited and noncurative in the majority of cases (Alexandrescu et al, 2005; Danson and Lorigan, 2005). According to the American Joint Committee on Cancer, patients with advanced metastatic melanoma (stage IV) have a 5-year survival rate of only $2 \%$ (Balch et al, 2001).

Increased signalling through the RAF/MEK/ERK pathway, as a result of autocrine stimulation by basic fibroblast growth factor and hepatocyte growth factor, is implicated in melanocytic tumorigenesis (tumour growth, invasion and metastasis) (Satyamoorthy et al, 2003). Furthermore, the activity of ERK, which is downstream of RAF, has been shown to increase from

\footnotetext{
*Correspondence: Dr T Eisen, Urology, Skin and Lung Units, The Royal Marsden Hospital, Sycamore House, Downs Road, Sutton, Surrey SMT 5PT, UK. E-mail: tim.eisen@icr.ac.uk
}

Received 10 April 2006; revised 27 June 2006; accepted 27 June 2006; published online I August 2006 early- to advanced-stage melanoma (Satyamoorthy et al, 2003). This increased ERK activity may be the consequence of activating $B R A F$ mutations, which are present in up to $80 \%$ of human melanomas (Davies et al, 2002; Chang et al, 2004; Garnett and Marais, 2004). The most prevalent oncogenic BRAF mutation is the V600E BRAF mutation (previous terminology, V599E), which is present in $63 \%$ of melanomas (Brose et al, 2002). The increased apoptosis, observed in human melanoma cell lines when $B R A F$ expression is downregulated using RNA interference, supports a role for oncogenic $B R A F$-driven MEK/ERK overactivation in maintaining the transformed phenotype of malignant melanoma cells (Hingorani et al, 2003; Karasarides et al, 2004). This observation also suggests that $B R A F$ is a rational target for the design of targeted agents to treat melanoma.

The orally administered targeted-agent sorafenib (Nexavar ${ }^{\circledR}$, Bayer Pharmaceuticals Corporation, West Haven, CT, USA) was originally developed as an inhibitor of the RAF serine/threonine kinases (RAF-1, wild-type BRAF, V600E BRAF) (Wilhelm et al, 2004). However, results of in vitro studies have since shown that 
sorafenib is a potent multikinase inhibitor, which also targets receptor tyrosine kinases associated with tumour angiogenesis (VEGFR-2, VEGFR-3, PDGFR- $\beta$ ) and tumour progression (c-KIT, FLT-3) (Wilhelm et al, 2004). Sorafenib has also been shown to inhibit the growth of several human tumour xenograft models by targeting tumour cell proliferation and/or endothelial cellmediated tumour angiogenesis (Wilhelm et al, 2004).

Sorafenib monotherapy has been shown to have a manageable side effect profile in Phase I/II/III studies (Strumberg et al, 2003, 2005; Ratain et al, 2004, 2006; Awada et al, 2005; Escudier et al, 2005). The most common toxicities associated with sorafenib are hand-foot skin reaction (HFS), rash and diarrhoea (Strumberg et al, 2003, 2005; Ratain et al, 2004, 2006; Awada et al, 2005; Escudier et al, 2005). However, these adverse events are predominantly mild to moderate in severity and easily manageable. The efficacy of sorafenib monotherapy in patients with advanced, refractory renal cell carcinoma (RCC) was first demonstrated in a Phase II randomised discontinuation trial (RDT), which showed a significantly longer progression-free survival (PFS) relative to placebo. Sorafenib received marketing approval in the US in December 2005 for the treatment of advanced RCC, based on the results of the RDT as well as the Phase III placebo-controlled TARGETs (Treatment Approaches in Renal cancer Global Evaluation Trial). The Phase III trial demonstrated a statistically significant doubling of PFS and longer overall survival (hazard ratio $=0.72$ for sorafenib over placebo) in patients treated with sorafenib relative to placebo treatment (Nexavar prescribing information, 2006) (Escudier et al, 2005).

Here, we present an analysis of the efficacy and safety of sorafenib monotherapy in a cohort of patients with progressive advanced melanoma.

\section{MATERIALS AND METHODS}

\section{Patients' characteristics and study design}

This Phase II, placebo-controlled RDT was conducted at five centres in two countries (four centres in the US, one centre in the UK). Enrolment began on 25 September 2002. All patients participating in the RDT provided written, informed consent. The trial protocol received institutional ethics committee approval at each participating centre, and was conducted in accordance with Good Clinical Practice guidelines and the Declaration of Helsinki.

The design of this RDT, including patients' details and inclusion and exclusion criteria, has been described previously (Ratain et al, 2006). Briefly, eligible patients had histologically or cytologically confirmed, progressive advanced unresectable, or metastatic cancer. Sorafenib was initially administered to all patients in a 12-week, open-label, run-in period using continuous oral dosing at $400 \mathrm{mg}$ twice daily (b.i.d.). After the 12-week run-in period, disease status was assessed based on change in bidimensional tumour measurements from baseline. Patients with $\geqslant 25 \%$ tumour shrinkage continued to receive sorafenib open label until they experienced disease progression or unacceptable toxicity, whereas patients with progressive disease $(P D ; \geqslant 25 \%$ tumour growth or other clinical evidence of progression) were discontinued. Those patients who had an unconfirmed change in tumour size of $<25 \%$ were randomised in a double-blind fashion to receive either sorafenib $400 \mathrm{mg}$ b.i.d., continuously, or matching placebo from week 12 onwards. Patients with progression of disease - defined as a change in bidimensional tumour measurement from randomisation of $\geqslant 25 \%$, or clinically assessed progression - at any time after randomisation, were unblinded. Progressors from the placebo group were given the opportunity to crossover to sorafenib, whereas those who progressed while on sorafenib were discontinued. In this analysis, we evaluated the efficacy and safety of sorafenib monotherapy in a cohort of patients with progressive advanced (ie unresectable or metastatic) melanoma, who participated in this Phase II RDT.

\section{Efficacy and safety assessments}

As described previously (Ratain et al, 2006), the primary end point was the percentage of patients remaining progression free at 12 weeks postrandomisation. Secondary endpoints included PFS after randomisation, overall PFS, tumour response rate and safety (Ratain et al, 2006). This analysis will focus on the changes from baseline in bidimensional tumour measurements after the run-in phase, tumour responses for the entire treatment period, PFS and safety findings for a cohort of patients with advanced melanoma.

Tumour response was assessed at 12 weeks, and every 6 weeks thereafter, using standard bidimensional measurements in accordance with WHO guidelines for partial response (PR), stable disease (SD) and PD. Objective responses (ie minor response and $\mathrm{PR}$ only) were confirmed at least 4 weeks after the original documentation.

Safety was assessed for the entire treatment period (run-in plus randomisation). All patients who received at least one dose of study drug were evaluable for safety. Safety assessments were performed every 3 weeks during the run-in and randomised phases, and every 4 weeks thereafter. Toxicities were graded according to the National Cancer Institute Common Toxicity Criteria version 2.0 (NCI-CTC v2.0), and relationship to study drug was recorded.

\section{Biomarkers: BRAF and RAS oncogenes}

DNA extracted from the tumour biopsies of patients was screened for the presence of oncogenic $B R A F$ and $R A S$ mutations. Owing to the majority of oncogenic $B R A F$ mutations in melanoma patients are likely to occur within the kinase domain (eg the prevalent V600E BRAF mutation), exon 15 was analysed first. Exon 11 of $B R A F$ was also screened for the presence of common mutations in the glycine-rich loop. In addition, exons 2 and 3 of NRAS (University of Pennsylvania and Royal Marsden Hospital Melanoma Unit) and KRAS (Royal Marsden Hospital Melanoma Unit) were screened for common oncogenic mutations. Fresh tumour samples, obtained from patients at The Royal Marsden Hospital Melanoma Unit and the University of Pennsylvania, were snapfrozen and stored at $-80^{\circ} \mathrm{C}$ until use. Genomic DNA from snapfrozen samples, or from paraffin-embedded blocks, was then extracted by lysing tumour samples with proteinase $\mathrm{K}$ and tissue lysis buffer, and treatment with RNAse to eliminate residual RNA. The extracted genomic DNA was then purified on a silica-gel membrane column. BRAF and RAS exons of interest were then amplified by the polymerase chain reaction (PCR) using the following primers, under optimised conditions:

Forward Reverse

BRAF exon 15: TCATAATGCTTGCTCTGATAGGA GGCCAAAAATTTAATCAGTGGA BRAF exon 11: TCCCTCTCAGGCATAAGGTAA CGAACAGTGAATATITCTTTGAT NRAS exon 2: GAACCAAATGGAAGGTCACA AACCTAAAACCAACTCTITCCCA KRAS exon 3: TCAAGTCCTTTGCCCATTTT TGCATGGCATTAGCAAAGAC

Polymerase chain reaction products were then purified by agarose gel electrophoresis, and automated dideoxy DNA sequencing was performed using the primers that were used for the amplification step and Big-Dye Terminator RR mix. DNA sequences were analysed using the Sequencher 4.2.1 programme. 


\section{Statistical analysis}

The PFS attributable to sorafenib was estimated by combining information from the various treatment groups and treatment periods. All patients contributed to the estimate of PFS for the first 12 weeks of therapy. The estimate of PFS for the first 12 weeks was combined with an estimate of PFS after 12 weeks, the latter assuming a patient was alive and progression free at 12 weeks. Progression-free survival was estimated after 12 weeks as a weighted average of group-specific PFS for the two groups (ie open-label and randomised groups). This methodology has been fully described previously (Ratain et al, 2006).

\section{RESULTS}

In total, 502 patients with a variety of tumour types, including RCC and melanoma, were enrolled in this RDT; 501 of these patients received sorafenib. This report focusses on the 37 treated patients who had progressive advanced melanoma at the time of study enrolment. The baseline characteristics of these patients are shown in Table 1. Forty-one percent of these patients had an Eastern Cooperative Oncology Group performance status (ECOG PS) of 0 , and $57 \%$ of patients had an ECOG PS of 1 . At baseline, $70 \%$ of these patients had failed at least one prior systemic therapy, and $27 \%$ had also received prior radiotherapy.

\section{Twelve-week response assessment}

The 12 -week sorafenib run-in phase was completed by 33 (89\%) of patients with advanced unresectable or metastatic melanoma. The

Table I Baseline characteristics for all treated patients with advanced melanoma

\begin{tabular}{|c|c|}
\hline Characteristics & Patients $(n=37)$ \\
\hline \multicolumn{2}{|l|}{ Gender (n (\%)) } \\
\hline Male & $23(62)$ \\
\hline Female & $14(38)$ \\
\hline Median age (years (range)) & $53(18-85)$ \\
\hline \multicolumn{2}{|l|}{ ECOG PS (n (\%)) } \\
\hline 0 & $15(4 \mid)$ \\
\hline I & $21(57)$ \\
\hline 2 & $0(0)$ \\
\hline 3 & I (3) \\
\hline \multicolumn{2}{|l|}{ AJCC stage at study entry (n (\%)) } \\
\hline III & $4(11)$ \\
\hline IV & $21(57)$ \\
\hline \multicolumn{2}{|l|}{ Most common sites of disease (all lesions) ${ }^{a}(n(\%))$} \\
\hline Lung & $24(65)$ \\
\hline Lymph node & $20(54)$ \\
\hline Liver & $12(32)$ \\
\hline Adrenal & $8(22)$ \\
\hline Median duration of disease ${ }^{b}$ (years (range)) & $2.7(0.2-12.9)$ \\
\hline \multicolumn{2}{|l|}{ Prior therapy (n (\%)) } \\
\hline Systemic anticancer therapy & $26(70)$ \\
\hline Surgery & $36(97)$ \\
\hline Radiotherapy & $10(27)$ \\
\hline \multicolumn{2}{|l|}{ Number of prior systemic regimens } \\
\hline None & II (30) \\
\hline 1 & $13(35)$ \\
\hline 2 & $8(22)$ \\
\hline$\geqslant 3$ & $5(13)$ \\
\hline
\end{tabular}

AJCC $=$ American Joint Committee on Cancer; ECOG PS = Eastern Cooperative Oncology Group performance status. ${ }^{a} \mathrm{All}$ target and nontarget lesions occurring with a frequency $\geqslant 20 \%$. ' $Y$ ears from initial diagnosis to first study treatment. four patients who discontinued before the 12-week assessment all withdrew because of adverse events: three had dermatological events of grade 3 in severity (skin toxicity, $n=1$; plantar-palmar erythema, $n=2$ ), one had a grade 4 cerebral embolic event.

At week 12, investigator-assessed bidimensional tumour measurements were available for $34(92 \%)$ of the 37 patients. One patient $(3 \%)$ achieved tumour shrinkage $\geqslant 25 \%$ compared with baseline and consequently continued with open-label sorafenib treatment. This patient had an overall duration of treatment of 16 weeks, and PFS of 15 weeks. This patient had three measurable lung metastases. The first lung metastasis showed $75 \%$ shrinkage after 106 days of treatment and $84 \%$ shrinkage after 136 days. The second metastatic lung lesion had shrunk by $80 \%$ after 106 days of treatment and had disappeared by 136 days. The third lung metastasis had completely disappeared after 106 days' treatment. Six patients $(16 \%)$ had tumour measurements that remained within $25 \%$ of baseline levels. The first patient had SD after 89 days of treatment; there was no change in tumour size until PD was proven after 194 days of treatment. The second patient had SD after 79 days' treatment; there was a $5.9 \%$ change in tumour size. After 115 days of treatment this patient was proven to have PD. However, at a confirmatory scan after 176 days this patient had SD again. After 428 days this patient was considered to have PD by clinical judgment, but a scan at 512 days revealed that the patient still had SD. The third patient developed SD after 85 days of treatment (24\% change in tumour size), was considered to have PD after 113 days (53\% tumour growth), but had re-stabilised after 184 days' treatment (9.9\% tumour shrinkage). This patient finally progressed after 249 days' treatment. The fourth patient had SD after 96 days' treatment ( $0.6 \%$ change in tumour size) and was still stable after 138 days (6.4\% tumour shrinkage). At 404 days this patient still had SD (4.6\% tumour shrinkage), but then progressed after 453 days. The fifth patient with SD had progressed after 81 days on treatment, while the sixth patient had SD after 84 days treatment (15\% tumour shrinkage), and at day 124 (8.8\% tumour growth) but had PD confirmed after two scans after $237(2.9 \%$ growth) and 321 days' (20.6\% growth) treatment.

The patients with SD at week-12 were then randomised: three received placebo and three received sorafenib. Twenty-seven patients $(73 \%)$ had PD (ie tumour growth $\geqslant 25 \%$ or other clinical evidence of progression), and were discontinued.

\section{Antitumour activity: randomised phase}

Although all three patients with melanoma randomised to sorafenib progressed at 12 weeks postrandomisation (24 weeks from initiation); only two were discontinued. The third patient was considered by the investigator to be deriving clinical benefit and was, therefore, continued on sorafenib monotherapy. At 12 weeks postrandomisation, all three melanoma patients who received placebo had progressed and, therefore, were crossed over to sorafenib monotherapy in accordance with the study protocol. After crossing over, the three patients had further disease progression at 11,15 and 22 weeks, respectively. The patient who progressed after 15 weeks remained on sorafenib for a total duration of 73 weeks because, in the opinion of the investigator, the patient was continuing to derive clinical benefit.

\section{Antitumour activity: entire treatment period}

At the end of the entire treatment period, the confirmed investigator-assessed best responses (WHO criteria) to sorafenib were $19 \%$ SD, $62 \%$ PD and $19 \%$ unevaluable. The median PFS for the entire treatment period, based on investigator-assessed data, was 11 weeks ( $n=32$; range 9-12 weeks). The six randomised melanoma patients with SD had overall PFS durations of 16 $(n=3), 18(n=1), 28(n=1)$ and $34(n=1)$ weeks. The median 
time to disease progression for all melanoma patients over the entire treatment period was 11 weeks ( $n=30$; range 9-13 weeks).

\section{Safety}

Safety was assessed across the entire treatment period for all 37 patients with advanced melanoma. The most common treatmentemergent adverse events, regardless of attribution, were fatigue (81\%); pain (73\%); gastrointestinal adverse events, including diarrhoea (51\%) and constipation (46\%); and dermatological reactions (dermatology/skin - other, 49\%; alopecia, 38\%; HFS, $35 \%)$. The majority of these events were NCI-CTC v2.0 grades $1-2$. For example, seven patients $(18.9 \%)$ had grade 1 alopecia and a further seven (18.9\%) had grade 2 alopecia; none had grade 3, 4 or 5 alopecia. Grade 3/4 hypertension was observed in $14 \%$ of patients. Serious adverse events were reported in $51 \%$ of the melanoma patients, and were attributed mostly to disease progression rather than study drug. Hypertension was not reported as a serious adverse event, and none of the melanoma patients discontinued therapy because of hypertension. Adverse events of any grade attributed by the investigator to study drug (ie drug-related adverse events) were reported in $89 \%$ of patients (Table 2). The most common drug-related adverse events among melanoma patients were dermatological (rash/desquamation, $51 \%$; HFS, 35\%; and alopecia, 35\%); gastrointestinal (diarrhoea, 32\%; and stomatitis/pharyngitis, $22 \%$ ); or constitutional (fatigue, $43 \%$ ) (Table 2). The majority of drug-related adverse events were grades $1-2$ in severity. In total, $8 \%$ of patients experienced serious drugrelated adverse events (all $\geqslant$ grade 3 ). No grade 4 drug-related adverse events were reported. Six patients required dose reductions because of drug-related dermatological adverse events. Dose

Table 2 Incidence of drug-related adverse events reported in $\geqslant 10 \%$ of all treated patients $(n=37)$

\begin{tabular}{|c|c|c|}
\hline Adverse event & $\begin{array}{c}\text { All grades } \\
n(\%)\end{array}$ & $\begin{array}{c}\text { Grade } 3^{\mathrm{a}} \\
n(\%)\end{array}$ \\
\hline Any event & $33(89.2)$ & $12(32.4)$ \\
\hline \multicolumn{3}{|l|}{ Cardiovascular } \\
\hline Hypertension & $6(16.2)$ & $5(13.5)$ \\
\hline \multicolumn{3}{|l|}{ Dermatology } \\
\hline Rash/desquamation & $19(51.4)$ & $2(5.4)$ \\
\hline Hand-foot skin reaction & $13(35.1)$ & $4(10.8)$ \\
\hline Alopecia & $13(35.1)$ & $0(0.0)$ \\
\hline Flushing & $4(10.8)$ & $0(0.0)$ \\
\hline Other & $12(32.4)$ & $0(0.0)$ \\
\hline \multicolumn{3}{|l|}{ Constitutional symptoms } \\
\hline Fatigue & $16(43.2)$ & $0(0.0)$ \\
\hline Weight loss & $6(16.2)$ & $0(0.0)$ \\
\hline \multicolumn{3}{|l|}{ Gastrointestinal } \\
\hline Diarrhoea & $12(32.4)$ & $0(0.0)$ \\
\hline Anorexia & $5(13.5)$ & $0(0.0)$ \\
\hline Stomatitis/pharyngitis (oral/pharyngeal) & $8(21.6)$ & $0(0.0)$ \\
\hline Nausea & $5(13.5)$ & $0(0.0)$ \\
\hline Other ${ }^{b}$ & $5(13.5)$ & $0(0.0)$ \\
\hline Constipation & $4(10.8)$ & $0(0.0)$ \\
\hline \multicolumn{3}{|l|}{ Neurology } \\
\hline Neuropathy - sensory & $5(13.5)$ & $0(0.0)$ \\
\hline
\end{tabular}

interruptions were reported in 12 patients, of which 10 were due to drug-related adverse events.

\section{Biomarkers}

DNA was successfully extracted and screened from 17 of 22 tumour biopsies. Six biopsies had oncogenic V600E BRAF mutations within exon 15 , while the remaining 11 had wild-type $B R A F$. Of the six V600E BRAF-positive tumour biopsies, four were obtained from patients with $\mathrm{PD}$, one from a patient with SD and one from a patient who was unevaluable for response. Only one wild-type $B R A F$-positive tumour biopsy was derived from a patient with SD, nine were from patients with $\mathrm{PD}$, and one from an unevaluable patient. BRAF mutational status data for 15 patients who were evaluable for response are shown in Figure 1. No oncogenic BRAF mutations were identified in exon 11 in any of the tumour biopsies evaluated, and only one oncogenic NRAS $(61 \mathrm{~K})$ mutation was detected. No other oncogenic NRAS or KRAS mutations were identified.

\section{DISCUSSION}

The efficacy and safety of sorafenib monotherapy were evaluated in patients with advanced melanoma, who participated in a large Phase II RDT involving patients with several advanced solid tumour types (Ratain et al, 2006). An analysis focussing on melanoma patients was performed in the light of evidence supporting a role for increased signalling through RAF/MEK/ ERK in the onset and progression of melanoma (Davies et al, 2002; Chang et al, 2004; Garnett and Marais, 2004), and the prevalence of oncogenic V600E BRAF mutations in melanoma biopsies (Brose et al, 2002). Recent preclinical evidence, showing that blocking V600E BRAF expression promotes apoptosis in human melanoma cells, provided a rationale for targeting signalling through RAF/ MEK/ERK in the treatment of melanoma (Hingorani et al, 2003; Karasarides et al, 2004). Despite this rationale, the data in this study at the $400 \mathrm{mg}$ b.i.d. dose studied were disappointing, and are most consistent with a conclusion that the drug has little or no activity as a single agent in this disease.

In human xenograft models, sorafenib has been shown to act on tumour cells to exert an antiproliferative effect, and on endothelial

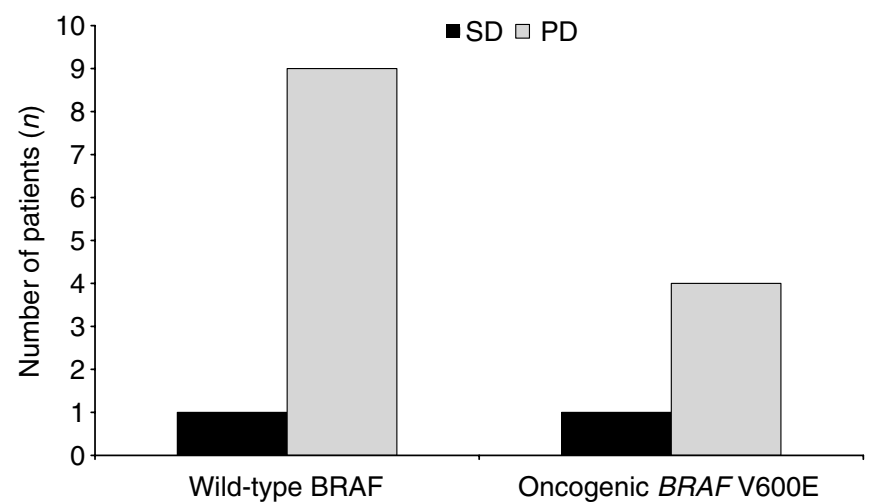

Figure I BRAF mutational status of advanced melanoma patients is not associated with disease status (status of I 5 patients evaluable for response). These patients were also evaluated for oncogenic BRAF mutations within exon II and oncogenic NRAS and KRAS mutations. One oncogenic NRAS (6IK) mutation was found. No other oncogenic NRAS or KRAS mutations were detected. Two patients were unevaluable for response $(n=I$ wildtype BRAF; $n=1$ BRAF V600E) and are not included in the above histogram. DNA could not be extracted from a further five biopsies. The mutational status of the biopsies from five patients was, therefore, not determined ( $n=3$ PD; $n=2$ SD). 
cells of the tumour vasculature to inhibit angiogenesis (Wilhelm et al, 2004). Sorafenib also induces apoptosis in several human cancer cell lines (Rahmani et al, 2005; Yu et al, 2005), including melanoma cells (Panka et al, 2006b). Sorafenib downregulates Mcl1 protein levels in a time- and dose-dependent manner to induce apoptosis in renal, colon and breast tumour lines (Rahmani et al, 2005; Yu et al, 2005). This effect involves enhanced proteasomal degradation of Mcl-1, which could be the consequence of RAF-1 inhibition. Finally, sorafenib induces caspase-independent apoptosis in A2058 and SKMEL5 melanoma cell lines (Panka et al, 2006a). However, the multiple molecular targets of sorafenib, and its dual effects on the tumour cell and the vascular endothelium, make it difficult to determine the mechanism of effect of this multikinase inhibitor in different tumour types, particularly in the absence of validated biomarkers.

In the present analysis, sorafenib monotherapy was well tolerated. Seven patients had PFS lasting between 16 and 34 weeks, although it is not clear whether or not this was an effect of sorafenib. Restabilisation of disease was also observed in one patient who was crossed over to sorafenib after progressing on placebo during the randomised 12-week treatment phase. This patient received sorafenib for a total of 73 weeks. There was no apparent relationship between the presence of an oncogenic V600E $B R A F$ mutation within exon 15 and the modest antitumour activity of sorafenib monotherapy. This latter observation is consistent with the lack of a clear relationship reported in another sorafenib trial (Eisen et al, 2005). Larger randomised clinical trials are ongoing and will more definitively explore the relationship between response and BRAF mutational status.

Despite showing little activity as a monotherapy in this RDT cohort, recently published observations suggest that sorafenib may enhance the antitumour activity of carboplatin and paclitaxel against melanoma (Flaherty et al, 2004). In an expanded Phase I study predominantly in melanoma patients, this combination induced one complete response $(<1 \%), 27$ PRs $(26 \%)$ and 61 SDs $(58 \%)$ in patients with advanced melanoma (Flaherty et al, 2004). It is possible that these findings may be due to sorafenib's inhibition of Raf kinase, a known mediator of taxane resistance (Britten and Klein, 2000). In another Phase I trial, the combination of sorafenib with dacarbazine induced a PR in three, and SD in five of 10 evaluable patients with advanced melanoma (Eisen et al, 2005). Further investigations are warranted to determine whether sorafenib may significantly increase response to chemotherapy and prolong PFS in patients with advanced melanoma.

Given the preclinical and clinical evidence supporting a role for oncogenic $B R A F$ in driving melanoma progression, it is unclear why sorafenib $400 \mathrm{mg}$ b.i.d. did not demonstrate significant activity as a monotherapy in advanced melanoma patients. It is conceivable that this lack of effect is due to insufficient concentrations of sorafenib being achieved within the plasma, and more importantly within the tumours of these patients. The $\mathrm{IC}_{50}$ of sorafenib in humans is approximately $5 \mu \mathrm{M}$ (Clark et al, 2005), and may not have been achieved in these melanoma patients. However, pharmacokinetic trials of sorafenib monotherapy have demonstrated steady-state concentrations consistently over the $\mathrm{IC}_{50}$ of $5 \mu \mathrm{m}$ for the recommended dosage of sorafenib (Clark et al, 2005). Therefore, further studies are required to investigate this possibility. It is plausible that proliferation of melanoma cells could be driven by an alternative signalling pathway, after signalling through RAF/MEK/ERK has been blocked. A further possibility is that a feedback mechanism could negate the effect of sorafenib in these melanoma patients. This contention is supported by the recent observation that sorafenib administration is associated with increased RAF-1 phosphorylation at Ser338 in human melanoma and other tumour cell types (Adnane et al, 2005). However, it remains to be determined whether such a feedback mechanism impairs clinical response to sorafenib monotherapy in melanomas with V600E BRAF mutations.

In conclusion, sorafenib is well tolerated but has little or no antitumour activity in advanced melanoma patients as a single agent at the dose evaluated ( $400 \mathrm{mg}$ b.i.d.). Ongoing trials in advanced melanoma are evaluating sorafenib combination therapies.

\section{REFERENCES}

Adnane L, Trail PA, Wilhelm S (2005) Sorafenib (BAY 43-9006) antagonizes Raf function not only by inhibiting Raf kinase activity but also by sequestering Raf protein into non-functional complexes. Presented at AACR-NCI-EORTC, November 2005, Philadelphia, PA

Alexandrescu DT, Dutcher JP, Wiernik PH (2005) Metastatic melanoma: is biochemotherapy the future? Med Oncol 22: 101-111

Awada A, Hendlisz A, Gil T, Bartholomeus S, Mano M, de Valeriola D, Strumberg D, Brendel E, Haase CG, Schwartz B, Piccart M (2005) Phase I safety and pharmacokinetics of BAY 43-9006 administered for 21 days on/7 days off in patients with advanced, refractory solid tumours. $\mathrm{Br} J$ Cancer 92: $1855-1861$

Balch CM, Soong SJ, Gershenwald JE, Thompson JF, Reintgen DS, Cascinelli N, Urist M, McMasters KM, Ross MI, Kirkwood JM, Atkins MB, Thompson JA, Coit DG, Byrd D, Desmond R, Zhang Y, Liu PY, Lyman GH, Morabito A (2001) Prognostic factors analysis of 17600 melanoma patients: validation of the American Joint Committee on Cancer Melanoma Staging System. J Clin Oncol 19: 3622-3634

Britten RA, Klein K (2000) Differential impact of Raf-1 kinase activity on tumor cell resistance to paclitaxel and docetaxel. Anticancer Drugs 11: $439-443$

Brose MS, Volpe P, Feldman M, Kumar M, Rishi I, Gerrero R, Einhorn E, Herlyn M, Minna J, Nicholson A, Roth JA, Albelda SM, Davies H, Cox C, Brignell G, Stephens P, Futreal PA, Wooster R, Stratton MR, Weber BL (2002) BRAF and RAS mutations in human lung cancer and melanoma. Cancer Res 62: 6997-7000

Chang DZ, Panageas KS, Osman I, Polsky D, Busam K, Chapman PB (2004) Clinical significance of BRAF mutations in metastatic melanoma. J Transl Med 2: 46
Clark JW, Eder JP, Ryan D, Lee R, Lenz H-J (2005) The safety and pharmacokinetics of the multi-targeted tyrosine kinase inhibitor (including Raf kinase and VEGF kinase), BAY 43-9006, in patients with advanced, refractory solid tumors. Clin Cancer Res 11: $5472-5480$

Danson S, Lorigan P (2005) Improving outcomes in advanced malignant melanoma: update on systemic therapy. Drugs 65: 733-743

Davies H, Bignell GR, Cox C, Stephens P, Edkins S, Clegg S, Teague J, Woffendin H, Garnett MJ, Bottomley W, Davis N, Dicks E, Ewing R, Floyd Y, Gray K, Hall S, Hawes R, Hughes J, Kosmidou V, Menzies A, Mould C, Parker A, Stevens C, Watt S, Hooper S, Wilson R, Jayatilake H, Gusterson BA, Cooper C, Shipley J, Hargrave D, Pritchard-Jones K, Maitland N, Chenevix-Trench G, Riggins GJ, Bigner DD, Palmieri G, Cossu A, Flanagan A, Nicholson A, Ho JW, Leung SY, Yuen ST, Weber BL, Seigler HF, Darrow TL, Paterson H, Marais R, Marshall CJ, Wooster R, Stratton MR, Futreal PA (2002) Mutations of the BRAF gene in human cancer. Nature 417: $949-954$

Eisen T, Ahmad T, Marais R, Gibbens I, James M, Affolter A, Chao D, Bergamini L, Schwartz B, Gore ME (2005) Phase I trial of sorafenib (BAY 43-9006) combined with dacarbazine (DTIC) in patients with metastatic melanoma. Eur J Cancer Suppl 3(2): 349

Escudier B, Szczylik C, Eisen T, Stadler WM, Schwartz B, Shan M, Bukowski RM (2005) Randomized phase III trial of the Raf kinase and VEGFR inhibitor sorafenib (BAY 43-9006) in patients with advanced renal cell carcinoma (RCC). J Clin Oncol 23(16_suppl): LBA4510 1-6-2005

Flaherty KT, Brose M, Schuchter L, Tuveson D, Lee R, Schwartz B, Lathia C, Weber B, O'Dwyer P (2004) Phase I/II trial of BAY 43-9006, carboplatin $(\mathrm{C})$ and paclitaxel $(\mathrm{P})$ demonstrates preliminary antitumor activity in the 
expansion cohort of patients with metastatic melanoma. J Clin Oncol 22(14S): 7507 (Abstract), 15-7-2004

Garnett MJ, Marais R (2004) Guilty as charged: B-RAF is a human oncogene. Cancer Cell 6: 313-319

Hingorani SR, Jacobetz MA, Robertson GP, Herlyn M, Tuveson DA (2003) Suppression of BRAF(V599E) in human melanoma abrogates transformation. Cancer Res 63: 5198-5202

Karasarides M, Chiloeches A, Hayward R, Niculescu-Duvaz D, Scanlon I, Friedlos F, Ogilvie L, Hedley D, Martin J, Marshall CJ, Springer CJ, Marais R (2004) B-RAF is a therapeutic target in melanoma. Oncogene 23: $6292-6298$

Panka DJ, Wang W, Atkins MB, Mier JW (2006b) The Raf inhibitor BAY 439006 (Sorafenib) induces caspase-independent apoptosis in melanoma cells. Cancer Res 66: $1611-1619$

Panka DJ, Wang W, Atkins MB, Mier JW (2006a) The Raf inhibitor BAY 43 9006 (Sorafenib) induces caspase-independent apoptosis in melanoma cells. Cancer Res 66: $1611-1619$

Rahmani M, Maynard Davis E, Bauer C, Dent P, Grant S (2005) Apoptosis induced by the kinase inhibitor BAY 43-9006 in human leukemia cells involves downregulation of mcl-1 through inhibition of translation. J Biol Chem 280: $35217-35227$

Ratain MJ, Eisen T, Stadler WM, Flaherty KT, Kaye SB, Rosner GL, Gore M, Desai AA, Patnaik A, Xiong HQ, Rowinsky E, Abbruzzese JL, Xia C, Simantov R, Schwartz B, O’Dwyer PJ. Related articles, links phase II placebo-controlled randomized discontinuation trial of sorafenib in patients with metastatic renal cell carcinoma. J Clin Oncol 2006; 24(16): $2505-2512$

Ratain MJ, Flaherty KT, Stadler WM, O’Dwyer P, Kaye S, Xiong H, Patnaik A, Gore M, Lee RJ, Eisen T (2004) Preliminary antitumor activity of BAY
43-9006 in metastatic renal cell carcinoma and other advanced refractory solid tumors in a phase II randomized discontinuation trial (RDT). J Clin Oncol 22: 381 (Abstract 4501). See also oral presentation at www. asco.org/

Satyamoorthy K, Li G, Gerrero MR, Brose MS, Volpe P, Weber BL, Van Belle P, Elder DE, Herlyn M (2003) Constitutive mitogen-activated protein kinase activation in melanoma is mediated by both BRAF mutations and autocrine growth factor stimulation. Cancer Res 63: 756 759

Strumberg D, Awada A, Piccart P, Hirte H, Moore M, Clark J, Lenz H-J, Lathia C, Schwartz B, Humphrey R (2003) Final report of the phase I clinical program of the novel raf kinase inhibitor BAY 43-9006 in patients with refractory solid tumors. J Clin Oncol 22: 203

Strumberg D, Richly H, Hilger RA, Schleucher N, Korfee S, Tewes M Faghih M, Brendel E, Voliotis D, Haase CG, Schwartz B, Awada A, Voigtmann R, Scheulen ME, Seeber S (2005) Phase I clinical and pharmacokinetic study of the novel Raf kinase and vascular endothelial growth factor receptor inhibitor BAY 43-9006 in patients with advanced refractory solid tumors. J Clin Oncol 23: 965 -972

Wilhelm SM, Carter C, Tang L, Wilkie D, McNabola A, Rong H, Chen C, Zhang X, Vincent P, McHugh M, Cao Y, Shujath J, Gawlak S, Eveleigh D, Rowley B, Liu L, Adnane L, Lynch M, Auclair D, Taylor I, Gedrich R, Voznesensky A, Riedl B, Post LE, Bollag G, Trail PA (2004) BAY 43-9006 exhibits broad spectrum oral anti-tumor activity and targets the Raf/ MEK/ERK pathway and receptor tyrosine kinases involved in tumor progression and angiogenesis. Cancer Res 64: 7099-7109

Yu C, Bruzek LM, Meng XW, Gores GJ, Carter CA, Kaufmann SH, Adjei AA (2005) The role of Mcl-1 downregulation in the proapoptotic activity of the multikinase inhibitor BAY 43-9006. Oncogene 24: 6861-6869 